\title{
La technique peut-elle tout résoudre ?*
}

\author{
Is technology capable of solving everything?
}

\author{
par Jean Dunglas \\ Conseil général du GREF
}

The ever-increasing population growth and the claim for a better standard of living, coupled with a water crisis in many parts of the world, require a new approach in managing water resources. Is technology capable of solving every aspect of the problem?

Many progresses have been made in the knowledge of resources and in water use efficiency. However, many water resources are polluted from the results of municipal, industrial and agricultural wastes. More often than not, we know the ways to avoid these adverse effects but there is lack of awareness by many decision-makers and financial allocations are continually shrinking.

We need to upgrade or replace existing dams and reservoirs and to build new dams to provide additional storage capacity. The financial and environmental cost of these projects is largely emphasized, but we know to foresee and prevent most of the negative impacts. Progresses in hydraulic machinery and structures, conveyance channels and conduits construction are important; so we have the capability of transfering large amount of water to another basin. However, in many cases, decisions making is blocked by the lack of political willpower or by ideological motives.

Cost of seawater desalinization and bad quality water treatments are continually decreasing but the processes require amount of low-priced energy and large investments.

The present know-how is very important but is often badly used. We need additional investments to new engineering design and improved hydrological tools, especially for pollution control and watershed management. Nevertheless, facing the population growth and climate changes, we need also new political long-term view and breakthrough in economic methods.

\section{I — PROGRÈS TECHNIQUES ET SOCIÉTÉ}

En cette fin du XXe siècle, l'humanité reste prise entre une croissance démographique encore assez largement incontrôlée dans divers pays du sud, et un intense besoin de progrès socioéconomique. Les pays en développement veulent plus de nourriture, de mieux-être, et, de façon générale, plus de biens de consommation. Les pays développés souhaitent plus de sécurité dans tous les domaines : emplois, environnement, risques majeurs, accidents climatiques. Dans tous les cas, les circonstances en jeu et les progrès désirés amèneront à disposer de façon régulière et à utiliser des quantités croissantes d'eau donc à en mobiliser les ressources correspondantes.'

Parallèlement, dans le monde entier les citoyens ont le sentiment que les techniques modernes, en constante progression, sont capables de résoudre tous ces problèmes : partout, quand on l'a voulu, les déserts ont été irrigués, les villes d'Arabie, et même les champs cultivés périurbains, sont alimentés en eau de mer dessalée, des barrages de plus en plus importants ont per-

1. R. Cans. la bataille de l'eau. Le Monde Editions, Paris, 1997 mis de créer des réserves gigantesques et les eaux usées des grandes villes des pays industriels sont maintenant traitées très convenablement. Quand un problème continue à se poser sans être résolu, le citoyen moyen y voit immédiatement une cause politique ou financière : la solution existe certainement mais les décisions nécessaires n'ont pas été prises, ou bien les sommes nécessaires pour les mettre en œuvre n'ont pas été obtenues.

Une analyse approfondie montre une réalité sensiblement plus complexe. Deux exemples en donnent un éclairage qui peut sembler paradoxal. [1]

En un siècle et demi la totalité de la population française a été progressivement alimentée en eau potable de bonne qualité, grâce au progrès constant des techniques hydrauliques (pompes, tuyaux...) et biochimiques (révolution pasteurienne, traitements physicochimiques...). Pourtant deux menaces nouvelles sont apparues sur les ressources, en premier lieu le risque de pollution accidentelle, essentiellement d'origine industrielle, imprévisible et aléatoire, et le risque de pollution diffuse provenant pour une part importante de l'agriculture

* Cet article est extrait du livre "L'eau en questions" paru aux éditions Romillat. 
intensive. Réglementation et normalisation n'ont pas encore apporté de réponses satisfaisantes à ces menaces.

Pendant la même période, l'installation dans la plupart des agglomérations de réseaux d'assainissement a permis l'évacuation, dans de bonnes conditions d'hygiène, des débits provenant des fortes précipitations. Mais la circulaire Caquot de 1947 imposait seulement la protection contre les crues décennales ; la circulaire Loriferne de 1976 laisse, quant à elle, le choix de la probabilité d'occurrence de la crue maximale à évacuer aux autorités locales. L'expérience a prouvé que, dans le cadre de la politique traditionnelle des collectivités locales, les élus hésitent face à ce choix. Les catastrophes de Nîmes en 1988 et celles de Vaison-la-Romaine en 1992 ont montré les difficultés qu'il y avait à vouloir gérer à la fois urbanisme et sécurité

Les progrès de la technique, la décision technique ellemême, sont en fait toujours étroitement dépendants des circonstances socio-économiques.

Les liens entre niveau technologique et développement des sociétés sont évidents et ont été maintes fois étudiés au plan historique. L'histoire humaine a été elle-même découpée en périodes caractérisées par une technique : paléolithique, néolithique, cuivre, bronze, fer... Le développement de la civilisation urbaine a été étroitement lié, entre autres, aux possibilités d'alimentation en eau et d'évacuation des déchets. La progression explosive du progrès technique, durant les cinq derniers siècles, peut d'ailleurs s'expliquer à la fois par des raisons scientifiques et socio-économiques.

Il est en effet clair que l'accroissement des connaissances de base est l'élément essentiel des progrès de la technologie ; celle-ci donne la possibilité, en retour, de construire les instruments et les outils qui alimentent le progrès scientifique. Plus la technologie est développée, plus elle permet des réalisations importantes pour un coût humain inférieur. C'est d'ailleurs en cela que la recherche scientifique est, à l'évidence, l'investissement le plus efficace qui existe, puisque les résultats qu'elle obtient sont ensuite utilisés par un grand nombre de générations.

Par ailleurs, à une échelle de temps plus modeste, les responsables politiques savent bien qu'une avance technique apporte toujours un avantage économique, militaire, ou social dans la concurrence internationale. Il est clair que les dépenses consenties pour la recherche le sont beaucoup plus pour cette raison qu'en vertu de considérations altruistes.

Cela étant, les réalisations technologiques d'une époque sont toujours à la fois le résultat d'un rêve (celui d'un dirigeant ou celui de toute une nation, parfois celui d'un simple citoyen), du savoir-faire existant dans le cadre d'un structure sociale appropriée, enfin de la quantité de travail humain qu'on est capable de mobiliser (soit par prélèvement monétaire, soit par une activité imposée ou forcée ou même librement acceptée).

A notre époque, comme aux précédentes, les possibilités de la technique sont strictement encadrées par ces trois conditions. Si l'une d'entre-elles fait défaut, il est impossible de réaliser.

\section{II $\square$ PEUT-ON TOUJOURS RÉSOUDRE LES PROBLÈMES LIÉS À L'EAU ?}

Ces problèmes ont fait l'objet de nombreuses publications en particulier dans le cadre de l'ouvrage collectif coordonné par J. Margat et J. R. Tiercelin [2]. Examinons maintenant de quelle manière l'extraordinaire arsenal mis à notre disposition par la science et la technique peut en fournir les solutions.

\subsection{Mieux connaître et mieux prévoir}

L'hydrologie, tant de surface que souterraine, et la climatologie ont beaucoup avancé depuis une dizaine d'années et les perspectives actuelles de recherche sont tout à fait considérables.

Les progrès en question sont liés au développement rapide de l'électronique, de l'informatique, de la télématique et des automatismes.

Les capteurs de surface, tant au niveau des quantités (pluies, débits, hauteurs d'eau, humidité du sol) que de la qualité (teneur en substances diverses) ainsi que ceux utilisés en hydrogéologie (essentiellement niveaux d'eau, débits et également qualité) sont devenus très fiables, automatiques, autonomes, interrogeables en temps réel et à distance.

Ces progrès doivent se poursuivre dans l'avenir par un accroissement sensible de l'autonomie, de la résistance aux agressions, et de la capacité de traitement et de télétransmission, ainsi que par un meilleur interfaçage.

D'autres progrès sont à attendre d'une utilisation plus intensive des données satellitaires, qui permettra d'accroître la connaissance des phénomènes tant au niveau de leur étendue que dans leurs détails (grâce aux capteurs infrarouges, à l'imagerie radar) ainsi que des radars météorologiques.

Il s'agit toutefois d'équipements coûteux, vieillissant assez vite (autant d'ailleurs par obsolescence technique que par usure vraie) et nécessitant un personnel de haut niveau ayant les capacités intellectuelles pour s'adapter très rapidement.

En France, EDF a pu organiser un remarquable système de mesure d'hydrologie de surface formant un réseau de 500 points d'instrumentation, totalement automatisé, travaillant en temps réel et ayant ainsi une excellente capacité de réaction en période de crise. (C. Cottereau, 1992)

Le traitement des informations recueillies permet de bénéficier quasi-instantanément du retour d'expérience sur la viabilité opérationnelle des modèles.

Il est clair que le handicap le plus important de ce type de système est son coût (installation, entretien, fonctionnement) et le nécessaire niveau scientifique du personnel. Pour l'instant, seuls des organismes ayant une puissante organisation et une large base financière (comme EDF) peuvent les mettre en place.

Pour traiter et interpréter les mesures, il faut des modèles de plus en plus performants.

En hydrologie de surface il existe deux grands types de modèles pluie-débit : les modèles "conceptuels" plutôt bâtis comme des séries de "boîtes noires" et les modèles "déterministes" qui cherchent à reproduire de la façon la plus précise possible les phénomènes physiques.

On sait d'autre part que la prévision météorologique progresse tant par les méthodes déterministes (précision à 5,7 et bientôt 10 jours) que par les méthodes probabilistes sur les tendances à plus long terme. [3]

EDF utilise couramment ces divers modèles et leur regroupement avec des modèles météorologiques de prévision des précipitations et des températures.

La mise en œuvre de modèles de ce genre pour la gestion d'ensembles hydrauliques importants en zone tropicale devient maintenant assez courante. (E. Servat et M. Sakho, 1992)

On constate ainsi que les progrès des outils et des méthodes s'accompagnent d'applications remarquables : mesures et modèles permettent de simuler et donc de suivre le fonctionnement de systèmes hydrauliques aménagés en vue de l'irriga- 
tion et de la production d'électricité. De telles simulations permettent de mieux appréhender, à l'avance, des situations critiques de pénurie et de s'y préparer en ce qui concerne la gestion des ouvrages.

La sophistication informatique et mathématique n'est évidemment pas un but en soi. Il faut avoir un outil dont la puissance croît régulièrement et qui permet la meilleure intégration entre l'hydrologie scientifique, l'hydrologie pratique et l'hydroclimatologie opérationnelle.

Des progrès de même nature existent en ce qui concerne le contrôle des aquifères. Certes, les nappes ne sont pas suivies avec autant de précision et d'ampleur que les eaux de surface ; toutefois les outils existent : capteurs de niveau et de pression, débits des exutoires, qualité des eaux, modèles de fonctionnement. L'usage plus intensif des eaux souterraines entraîne d'ailleurs, obligatoirement, l'extension de ce type de contrôle indispensable à une gestion rationnelle.

Parmi toutes ces avancées observons plus particulièrement celles qui concernent les modèles.

Elles ont suivi régulièrement l'augmentation rapide de la puissance des ordinateurs, qui s'est poursuivie à coût décroissant que ce soit en hydraulique de surface, en écoulement transitoire comme en transport solide, ainsi qu'en hydraulique souterraine, en simulation des pollutions à l'échelle d'un bassin entier, en représentation des paramètres écologiques (méthode de microhabitats p.ex.). Les progrès récents ont été impressionnants et rien ne s'oppose à ce qu'ils se poursuivent au même rythme.

Le développement des systèmes d'information géographique, qui consistent à rassembler dans des bases de données informatiques les caractéristiques topographiques et divers caractères thématiques d'un territoire, accroît encore, à un degré supérieur, le potentiel des méthodes de simulation, tous les types de modèles pouvant s'y rattacher.

Non seulement ces outils permettent une meilleure connaissance et un meilleur suivi, mais ils donnent également la possibilité de beaucoup mieux prévoir les effets et impacts des changements et des aménagements.

L'un des exemples les plus typiques concerne la modélisation des conséquences de l'augmentation des teneurs en gaz à effet de serre dans l'atmosphère, qui permet d'évaluer l'évolution des températures et du régime des pluies, de façon certes encore grossière à l'échelle régionale, mais néanmoins tout à fait révélatrice des grandes tendances [4], (J.C. Duplessy et P. Morel, 1990).

Mais il est nécessaire de progresser parallèlement en écologie générale et appliquée. Le cycle de l'eau est un des éléments fondamentaux du fonctionnement des écosystèmes et donc de l'écologie d'une zone, d'un continent et a fortiori de la planète. La question de la sensibilité des écosystèmes ou des groupes d'écosystèmes à une modification des conditions de transfert de l'eau revêt une grande importance pour évaluer l'impact des aménagements hydrauliques, des changements des conditions d'écoulement, des diverses pollutions.

Il s'agit malheureusement d'un domaine de recherche qui n'est que faiblement doté en moyens. Signalons, dans ce contexte d'insuffisance des connaissances, l'intérêt d'expériences comme celles qui ont été menées aux États-Unis dans l'Arizona. Sous le nom générique de "Biosphère II", elles mettent en jeu une structure complètement fermée (sauf au transfert d'énergie), avec des moyens jusque là inégalés (capteurs, informatique) de suivi et de contrôle, et renfermant plusieurs dizaines de groupes d'écosystèmes. [5] Fortement critiquées au plan scientifique, en particulier en ce qui concerne le manque de maîtrise sur la microbiologie du sol et des eaux, des expériences de ce genre sont néanmoins susceptibles d'apporter beaucoup sur la stabilité intrinsèque des écosystèmes, soit entièrement livrés à eux-mêmes, soit régulés artificiellement.

\subsection{Mieux faire, mieux réaliser}

A l'échelle du globe il existe assez peu de zones sèches ou désertiques qu'on ne puisse alimenter en eau à partir de prélèvements dans des ressources de surface ou souterraines plus ou moins lointaines, au moyen d'un équipement adapté. Compte-tenu du savoir faire actuel, le problème se situe essentiellement au niveau des investissements nécessaires et de la capacité locale à en mobiliser le montant.

Il s'agit alors de minimiser cet investissement :

- en économisant l'eau au transport et à la consommation ;

- en optimisant son utilisation ;

- en mobilisant éventuellement les ressources nouvelles nécessaires.

Il faut bien voir que cette problématique se place à la fois au plan de la quantité et à celui de la qualité.

\subsubsection{Consommation nette et qualité}

Il est nécessaire de préciser la notion de consommation de l'eau. Il n'y a, au-delà de la distinction classique entre consommation nette et prélèvement, vraiment consommation que lorsque, après usage, l'eau n'est plus utilisable ailleurs, le long de la partie liquide de son cycle. C'est le cas de l'eau intégrée à la production, ou de l'eau évaporée, qui représente la quasitotalité de l'eau consommée par les plantes. Mais en irrigation, en particulier en irrigation de surface, ou gravitaire, une partie de l'eau passe dans les nappes souterraines. Elle peut alors être soit en partie réutilisable à l'aval (cas des nappes alluviales du Nil, ou à un moindre degré celui de la nappe alluviale de la Crau), soit au contraire partiellement ou totalement perdue (nappes salées, nappes profondes). Mais il peut y avoir aussi une consommation supérieure au prélèvement quand l'eau rejetée est polluée. On compte alors soit sur la dilution (cas du $\mathrm{NaCl}$ dans le Rhin) soit sur les écosystèmes de la rivière pour atténuer ou éliminer cette pollution. La consommation réelle est, dans ce cas, bien supérieure à la quantité strictement prélevée, l'eau brute de la rivière devenant inutilisable, telle quelle, à l'aval sur une zone plus ou moins importante de son cours.

Quantité et qualité sont donc indissociables dans le raisonnement. Malheureusement, peu de prospectives de consommation prennent cet aspect en compte.

\subsubsection{Consommation et investissement}

Classiquement, pour satisfaire un besoin, on cherche une ressource la plus proche possible, on met en place un traitement si besoin est, puis, après usage, les eaux résiduaires sont rejetées en ayant subi éventuellement un nouveau traitement, le moins onéreux possible, en fonction des réglementations en vigueur. En l'absence de normes strictes de rejet, l'utilisateur calcule, bien entendu au plus juste, son installation et n'intègre évidemment pas dans ses calculs économiques les coûts externes imposés aux usagers aval par ses activités.

On touche là, très clairement, à un point tout à fait fondamental, à savoir l'incapacité des méthodes économiques actuelles à intégrer dans leurs bilans les externalités écologiques liées à l'usage de biens communs comme l'eau ou l'air. Or les décisions d'investissement sont prises en fonction d'in- 
dices économiques classiques ; elles aboutissent, dans ces conditions, à des situations toujours difficiles, souvent conflictuelles, et amènent la collectivité à des dépenses finalement beaucoup plus importantes. Des législations adaptées peuvent corriger partiellement ces travers ; elles ne sauraient, en aucun cas, avoir l'efficacité de mécanismes économiques moins primitifs que ceux en cours actuellement, et qu'il reste à mettre au point.

\subsection{Moins consommer, c'est moins prélever en utili- sant moins d'eau, mais aussi moins polluer}

Les ressources en eau à prix acceptable sont extrêmement variables suivant les zones considérées de la planète. Même là où l'eau est abondante, on a souvent intérêt, au sens le plus strict de l'économie classique, à réduire les consommations ne serait-ce que pour éviter les conflits d'usage pouvant apparaître dans l'avenir.

\subsection{1. Économie dans l'industrie}

Les économies possibles, dans le domaine industriel, sont considérables, quoique variables suivant les différents processus envisagés. De façon très grossière on peut dire que l'eau sert :

- à refroidir, par échange thermique ou évaporation (elle est aussi utilisée comme élément thermodynamique) ;

- à laver des produits bruts ou élaborés ou des zones de fabrication :

- dans les processus eux-mêmes, comme vecteur de produits solubles, de substances en suspension, ou comme élément de circulation intégré dans la fabrication en question.

Au niveau du refroidissement, la simple substitution, par des méthodes utilisant directement l'évaporation ou par l'usage de l'eau de mer ou de l'air, donne un potentiel d'économies considérable.

Dans les processus de lavage, les techniques utilisant les hautes pressions et les vibrations (soniques et ultrasoniques) permettent des diminutions appréciables de consommation.

Mais là encore, comme dans les processus de fabrication, il est clair que les progrès ne peuvent venir que de l'amélioration des méthodes de production elles-mêmes, en l'associant, bien entendu, au traitement et au recyclage de l'eau utilisée.

Bien souvent d'ailleurs, le bilan économique strict est amélioré car un certain nombre de produits récupérés sont utilisables. L'exemple classique dans ce domaine est celui des eaux de fromagerie dans lesquelles les résidus organiques (en particulier le lactose) servent de substrat à la fabrication de levures qui se commercialisent aisément.

Le potentiel de progrès technique est, dans ce domaine, quasiment sans limites autres qu'économiques.

Pour accélérer l'évolution, la méthode la plus efficace consiste probablement à taxer progressivement les rejets pour orienter dans le bon sens le bilan économique.

Pour ce qui concerne l'industrie, la voie du progrès est tout à fait claire : recycler de plus en plus, récupérer les substances polluantes, rejeter au minimum les produits toxiques. Il faut garder à l'esprit que le traitement de l'eau est toujours relativement facile dans le cycle de fabrication car il est ponctuel, porte sur des quantités limitées et sur des concentrations fortes. En revanche, une fois le processus de dilution amorcé, dans l'usine puis dans le milieu naturel, les remèdes sont de plus en plus coûteux et deviennent ensuite pratiquement impossibles à mettre en œuvre.

Cela étant, on peut dire que c'est justement ces zones et ces périodes de concentration intermédiaires dans l'environnement qui sont les plus dangereuses car, ensuite, la dilution ou la métabolisation naturelle écarte tout danger (situation schématisée à la figure 1).

Toutefois, même dans des cas très simples, la zone polluée peut être importante et les inconvénients ou dégâts tout à fait significatifs. Un très bon exemple est celui de la pollution de la nappe d'Alsace et du Rhin par le chlorure de sodium des mines de potasse.

Les dégâts causés aux zones touchées de la nappe, qui représentent plusieurs $\mathrm{km}^{2}$ sont importants et pratiquement irréversibles à moyen ou long terme. La pollution durera bien au-delà de l'arrêt de l'activité industrielle.

La pollution du fleuve Rhin lui-même est moins grave, car bien plus limitée dans le temps. Elle s'étend tout de même jusqu'à l'embouchure et entraine des inconvénients qui ne sont pas tous négligeables pour les Pays-Bas.

En ce qui concerne les centrales électriques de type thermique (classiques ou nucléaires) les principaux progrès à attendre viennent de l'amélioration du rendement des tours de réfrigération atmosphérique et du recours à l'utilisation de l'eau de mer (installation des centrales le long des côtes).

A moyen terme on peut compter sur un accroissement des rendements énergétiques grâce à des cycles thermodynamiques utilisant des sources chaudes à plus hautes températures. A plus longue échéance on peut espérer le développement massif de la production d'électricité à partir de cellules photovoltaïques ou de l'énergie éolienne.

\subsection{2. Économies dans l'agriculture (L. Rieul, 1993)}

Le problème des économies d'eau en irrigation est très ancien et a été traité dans de nombreuses publications. On peut dire, de façon très schématique, que le potentiel d'économie, en particulier à partir d'irrigations gravitaires de surface, est considérable tant en ce qui concerne l'utilisation de l'eau à la parcelle que son transport (passage à des canaux revêtus et aux canalisations sous pression, régulation fine des débits en fonction des besoins). Parallèlement, l'augmentation prévue de la popu-

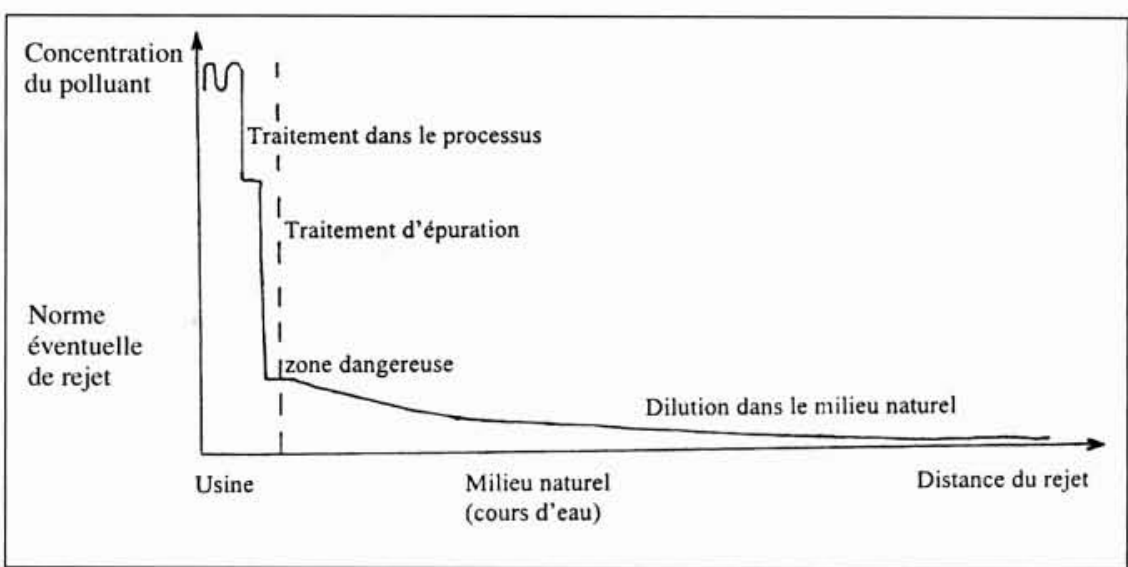

1. Évolution de la concentration d'un polluant à la sortie d'une installation industrielle. 
lation accroitra les besoins. De tout cela il ressort une conclusion assez évidente : on peut économiser de façon massive en améliorant les méthodes gravitaires et en passant à chaque fois que cela est possible à l'aspersion ou à l'irrigation localisée tout en utilisant le transport sous pression. Toutefois cela demande des investissements importants et surtout de larges disponibilités en énergie à un coût suffisamment bas. Là encore économies d'eau et électricité bon marché sont étroitement liées.

Les problèmes de pollution sont aussi aigus en agriculture que dans l'industrie mais ils en diffèrent par l'aspect diffus d'une part importante de ces pollutions.

Elles sont de diverses natures :

- matières organiques provenant en général d'installations d'élevage ou d'activités particulières à la ferme, ou encore de prétraitement de produits végétaux avant livraison à une industrie agro-alimentaire ; une part importante est constituée par des déjection animales ;

- biologiques, c'est-à-dire, parasites, bactéries, virus provenant, là encore, essentiellement d'installations d'élevage ; bien que non négligeable ce type de pollution n'a pas l'ampleur de celle provenant des collectivités ou des centres hospitaliers ;

- engrais ; il s'agit essentiellement de l'azote sous forme ammoniacale ou de nitrate et dans une moindre mesure de potasse ou de phosphore (essentiellement sous forme de phosphates). La pollution par les phosphates est en grande partie responsable des problèmes d'eutrophisation. Toutefois une part importante du phosphore soluble retrouvé dans les eaux provient des rejets urbains (lessives) ;

- substances phytosanitaires : elles font l'objet d'une surveillance attentive car même présents à de faibles concentrations, ces produits sont très actifs et le plus souvent dangereux.

- substances minérales particulières (hydrocarbures, certains sels...) ou parfois métaux lourds (associés à des engrais minéraux).

En agriculture intensive, la menace la plus sérieuse provient de l'azote (nitrates), de substances phytosanitaires, dans une moindre mesure de la matière organique.

Les problèmes dus aux pollutions agricoles alimentent bien des débats : l'agriculture intensive est considérée comme particulièrement polluante et on lui oppose les vertus supposées de l'extensification.

Certaines études (menées en particulier aux États-Unis et en Allemagne) sont plus nuancées. Il n'est pas du tout sûr en effet, que ramenées à l'unité de produit agricole, les méthodes extensives soient moins polluantes. Il s'agit surtout d'une question de dilution (laquelle néanmoins joue un rôle important comme dans le cas des nitrates pour lesquels la réglementation européenne limite les teneurs dans les eaux brutes).

D'importantes recherches sont menées pour réduire ces pollutions et des résultats substantiels ont déjà été atteints. De très grands progrès sont possibles : nouveaux itinéraires techniques minimisant le passage des substances dans les milieux, matériel optimisant et donc diminuant les quantités épandues, produits phytosanitaires biodégradables et, à moyen ou long termes, plantes transgéniques fixant l'azote de l'air et capables de résister aux attaques des maladies et parasites.

Une autre voie est celle de l'agriculture hyperintensive en milieu complètement fermé (serres) avec recyclage complet des effluents. Il est tout à fait possible, qu'en production végétale, ce type de production devienne assez vite économiquement viable, surtout si des formes adaptées de taxation des pollutions diffuses sont mises en place.
En production animale hors-sol, il est clair qu'il faudra arriver très rapidement à des méthodes permettant soit le traitement partiel ou total des lisiers sur place, soit leur transport permettant un épandage dans de bonnes conditions sur des sols agricoles. De grands progrès sont possibles dans ce domaine, mais les recherches pour y parvenir ne sont, à l'évidence, pas suffisamment poussées. Là encore un mécanisme économique est à mettre au point pour stimuler ce développement.

\subsection{3. Économies dans les collectivités - Importance de la collecte et du traitement des eaux usées}

Les consommations d'eau sont extrêmement variables suivant qu'il s'agit de milieu urbain ou rural, de pays industrialisés ou en développement, de zones riches en ressources en eau ou non, enfin du prix local de l'énergie.

Des évaluations ont été faites, par agrégats géographiques, avec des essais de prospective, par divers auteurs (voir en particulier [1]). Les hypothèses prises en compte sont toutes, bien sûr, discutables (tant en ce qui concerne l'évolution démographique que celle des consommations unitaires). Ces études ont l'avantage de donner toutefois un ordre de grandeur rationnel.

Cela étant, les progrès à attendre ont trait :

- aux consommations individuelles ;

- aux structures du transport et de la distribution ;

- à la réduction de l'usage de substances polluantes ;

- au traitement des eaux usées.

Dès qu'il s'agit de branchements particuliers, la régulation de la consommation individuelle ne se fait efficacement que par une tarification adaptée. Il s'agit essentiellement d'un problème de politique locale. La technique peut permettre de progresser en proposant des machines ou installations individuelles plus économes mais, là encore, la seule incitation vraiment efficace reste monétaire d'autant qu'elle permet également de lutter contre les fuites chez les particuliers.

Les structures de transport et de distribution doivent être adaptées à cette exigence. Il y a beaucoup à attendre des progrès en automatisme et en télématique.

Dans bien des pays en développement, les fuites dans les réseaux urbains restent considérables.

Elles entraînent des dangers sanitaires évidents et sont funestes pour l'équilibre financier des compagnies de distribution. La remise en état des réseaux d'eau potable est souvent le premier acte vraiment efficace d'économie qu'il est possible de réaliser. Rappelons à cet égard que par une politique rationnelle d'entretien et de reprise, le rendement du réseau de distribution de Paris est passé en 25 ans d'environ $50 \%$ à près de $90 \%$.

Le problème des eaux usées, de leur collecte et de leur traitement est encore plus aigu, surtout dans le Tiers-Monde. La pollution des collectivités est essentiellement de nature organique, biologique, (bactéries, virus, parasites) et chimique (hydrocarbures, détergents, phosphore). A l'échelle mondiale, le nombre de stations d'épuration efficaces est encore dramatiquement insuffisant ; beaucoup de rivières et même de fleuves deviennent simplement le grand égout des agglomérations qui les bordent. L'état des réseaux de collecte, quand ils existent, est souvent déplorable, les fuites étant fréquentes, avec de gros risques de contamination du réseau d'alimentation en eau.

Rappelons que, même dans un pays développé comme la France, bien des collectivités ne sont pas encore équipées de stations d'épuration.

Les progrès techniques dans ce domaine sont continus et le potentiel de développement reste considérable. De petites 
révolutions sont susceptibles de se produire à moyen ou long terme, comme la mise en place de prétraitement, par immeuble ou groupe d'immeubles.

Les principales difficultés, dans les pays industrialisés, viennent de l'existence de réseaux non séparatifs, de rejets d'industries mal contrôlés et des variations saisonnières de populations (tourisme). Cela est parfaitement maîtrisable et ne dépend que de décisions politiques et du volume des investissements disponibles.

Dans les pays en développement, la croissance beaucoup trop rapide des mégapoles induit en bien des endroits des situations sanitaires catastrophiques qui ne peuvent être améliorées que par des décisions très volontaristes à un coût financier élevé. Il faut bien garder à l'esprit qu'il s'agit là d'un des plus grands problèmes posés par l'accroissement trop rapide des populations et leur entassement autour des grandes cités. L'analyse des origines de ces concentrations excessives sortirait du cadre de cette étude. On peut toutefois citer l'une d'entre elles, parmi les plus importantes, à savoir l'exode rural dont l'une des causes est la ruine des petits paysans, causée par un commerce mondial des produits alimentaires où une concurrence excessive conduit à des prix de marché trop bas.

Quoi qu'il en soit, si les équipements nécessaires ne sont pas mis en place, ces agglomérations en croissance constante transformeront rivières, fleuves, lacs, et bordures maritimes en véritable cloaques, pouvant amener rapidement à l'effondrement des écosystèmes locaux et régionaux incapables de supporter une telle surcharge. Or un écosystème détruit n'est pas du tout aisé à reconstituer.

Ce problème est peut-être aussi important que celui de la faim, car il est beaucoup moins facile de traiter ou de recycler les eaux usées que d'importer de la nourriture par le commerce ou l'aide internationale.

Il existe un problème annexe qui ne peut être abordé ici, mais qu'il convient de signaler, à savoir l'évacuation des eaux de pluies et les risques d'inondations. Il s'agit là de risques aléatoires à traiter, à l'échelle d'un bassin versant, par des politiques d'urbanisme et des techniques appropriées.

Rappelons à cet égard, que toute inondation urbaine brutale entraîne des phénomènes de pollution importants des rivières et même des nappes superficielles.

\subsubsection{Les trois stades du traitement des pollutions}

Finalement, que ce soit dans le domaine industriel, agricole ou dans celui des collectivités, on se trouve en présence de trois types de situations.

- Laisser le soin à la dilution et aux écosystèmes d'épurer rejets et effluents. C'est en gros la situation actuelle pour une part importante de l'agriculture et une proportion variable des collectivités.

- Agir sur les processus et itinéraires techniques pour minimiser pertes et effluents et, quand cela est possible, effectuer des traitements plus ou moins importants en éliminant les substances les plus gênantes pour ne pas trop surcharger le milieu naturel. C'est la principale voie de progrès actuelle. Elle demande, pour être menée rationnellement, une bonne connaissance de la stabilité des écosystèmes en face de ces surcharges.

- Travailler au maximum en circuit fermé en recyclant tout ce qui est possible. C'est à l'évidence la voie de l'avenir. Elle est déjà, dans certains cas limités, financièrement viable. Elle demande beaucoup de progrès et de développement technique et surtout l'adoption des nouvelles méthodes de calculs économiques per- mettant d'imputer au consommateur final (donc au producteur) les coûts externes et les coûts induits, dans le futur, par l'installation.

Elle nécessitera, de ce fait, des changements sensibles au niveau des mentalités et des comportements sociaux, tant au niveau des populations que des élus et décideurs privés.

\subsection{Mieux mobiliser les ressources - Mobiliser de nouvelles ressources}

Il s'agit tout d'abord d'améliorer les méthodes de stockage et de régulation des eaux de surface ainsi que de gestion et d'exploitation des aquiferes, en évitant en particulier les effets nocifs annexes.

Il s'agit ensuite d'améliorer les méthodes de transport.

Il s'agit enfin de mobiliser, dans les meilleures conditions, les ressources que les progrès des techniques rendent maintenant utilisables.

\subsubsection{Eaux de surface}

Les deux méthodes classiques d'exploitation des eaux de surface sont les pompages en rivière (associés à de petits barrages de régulation de niveau) et les barrages réservoirs proprement dits.

Les impacts négatifs de ces équipements sont souvent détaillés avec complaisance. Voyons dans quelle mesure ces inconvénients sont maîtrisables.

\subsubsection{Evaporation des retenues}

Elle est difficile à éviter. Les diverses tentatives visant à la diminuer par le dépôt de films chimiques (hydrocarbures, silicones) ou de couches de microbilles de plastique, ou même de feuilles flottant en surface ont été, pour l'instant et dans la plupart de cas, des échecs ou des succès limités. La seule solution vraiment efficace est de diminuer l'importance relative de l'évaporation en utilisant des retenues les plus profondes possibles. Une autre réponse est d'utiliser des stockages dans des aquifères souterrains, même artificiels.

\subsubsection{Comblement des retenues et épuisement des sites de} barrages (F. Combes et M. Meunier, 1992)

Quatre types de solutions sont possibles :

- Au niveau du barrage et de la retenue elle-même, prévoir l'évacuation des sédiments au fur et à mesure de leur arrivée, spécialement en période de crue, par des dispositifs appropriés c'est-à-dire des vannes associées à des courants de densité. Ce type d'équipement, quoique techniquement difficile, a été réalisé avec succès. Il nécessite d'importantes études préalables sur modèles. Du fait de ces difficultés il est malheureusement encore peu pratiqué.

- Vidanger les sédiments déjà déposés, par des chasses de fond ou bien par aspiration ou par dragage. Ces solutions ne sont que partielles. Les chasses sont polluantes pour l'aval ; les dragages sont très coûteux.

- Piéger les sédiments à l'amont. Là encore la solution n'est que partielle. Évacuer ces sédiments reste coûteux, voire même dans beaucoup de cas, quasiment impossible et l'équilibre érosion-dépôt, à laval, est rompu.

- Limiter l'érosion et les transports solides. Les techniques sont bien connues (restauration des terrains en montagne) et efficaces ; les bénéfices annexes peuvent être considérables (conservation des sols). La méthode n'arrête toutefois pas complètement l'érosion et les dépôts.

Les ingénieurs d'EDF affirment pouvoir, en jouant sur ces quatre méthodes, stabiliser complètement le phénomène. 
L'efficacité n'est sans doute pas aussi grande sous des climats moins favorables qu'en France, et il est probable que de nombreux sites de barrages seront, d'ici quelques décennies ou quelques siècles, stérilisés.

Il est évident que dans tous les nouveaux équipements, les techniques de protection correspondantes devraient être mises en œuvre, ce qui est bien loin d'être encore le cas (toujours pour satisfaire les critères de rentabilité à court terme).

\subsubsection{Eutrophisation des retenues}

Elle est maîtrisable par la diminution des pollutions agricoles et le traitement des rejets des collectivités.

\subsubsection{Réduction des débits des cours d'eau en aval des dérivations}

Les cas du Nil, du Rio Grande et de l'Euphrate sont cités dans [1]. Celui de l'Amou Daria et du Syr Daria, entraînant la réduction de la surface de la mer d'Aral, a été abondamment repris par les médias.

De tels problèmes ne peuvent être résolus que par une diminution des consommations ou de coûteux transferts inter-bassins. Notons qu'ils sont toujours prévisibles et que leur non prise en compte n'est pas excusable.

\subsubsection{Impacts de l'exploitation des barrages}

Ces ouvrages représentent, par leur seul présence, un changement considérable dans les mécanismes d'écoulement tant en ce qui concerne les eaux de surface que les nappes alluviales associées.

Même une gestion purement hydro-électrique, donc à but strictement énergétique, amène des modifications parfois très importantes du régime des eaux, ce qui influence évidemment toutes les conditions hydro-écologiques à l'aval et peut perturber aussi la satisfaction des besoins des usagers.

Il s'y ajoute une transformation des phénomènes de transport, de dépôt et de reprise des sédiments. La modification de ces mécanismes aboutit fréquemment à des changements de niveau des nappes alluviales à l'aval qui peuvent, dans certains cas, avoir des effets désastreux.

Il n'y a pas de solutions toutes faites à ces types de problèmes.

La seule manière d'éviter, autant que possible, les impacts négatifs consiste à modéliser soigneusement, à l'avance, le système formé par l'ouvrage et les tronçons de rivières intéressées, de manière à en prévoir, du mieux possible, les effets négatifs et $\mathrm{y}$ apporter en même temps les remèdes nécessaires.

Le savoir-faire actuel permet, on l'a vu, ce genre de prévision même si pour certains aspects (évolution géomorphologique, transports de sédiments) on peut seulement n'en avoir qu'un aperçu approximatif. Ces modélisations doivent être intégrées dans les études préalables de manière à envisager, dès le départ, les mesures correctives éventuelles et établir le vrai bilan économique en conséquence.

On peut, certainement, aller bien plus loin encore dans ce domaine, et un renforcement de la recherche y est éminemment souhaitable.

En matière de barrage proprement dit, beaucoup de progrès sont possibles.

L'innovation la plus importante, au niveau des matériaux, dans ces dernières années, a été la généralisation de l'utilisation du béton compacté au rouleau (BCR) qui autorise d'intéressantes diminutions de prix de revient.

La mise au point de bétons spéciaux beaucoup plus résis- tants que les bétons classiques, tant en compression qu'en traction, n'a pas encore amené, semble-t-il, les concepteurs à en prévoir l'utilisation, sauf en des points très limités, dans les ouvrages hydrauliques.

L'apparition régulière sur le marché de nouveaux plastomères et élastomères toujours améliorés, conduira forcément à les utiliser dans les organes d'étanchéité, et l'on notera avec intérêt la réapparition en applications hydrauliques des structures gonflables.

Les progrès en matière de conception et de calculs sont continus; ils bénéficient de l'accroissement considérable de la puissance des ordinateurs et de l'avance prise en matière de $\mathrm{CAO}$ et de DAO en construction et en mécanique.

On est maintenant capable, en hydrologie opérationnelle, de gérer avec le maximum d'efficacité et en temps réel les flux et les stocks hydrauliques en fonction de la ressource et de la demande. Les calculs de remplissage et de crue de projet bénéficieront évidemment des progrès réalisés. La sûreté des ouvrages s'en trouvera largement améliorée.

Malgré les coûts non négligeables de l'instrumentation ainsi que des systèmes de transmission et de traitement, un nombre croissant de barrages existants devraient, dans les années à venir, bénéficier des nouvelles méthodes de gestion-prévision en temps réel. Cela contribuera encore mieux à les assurer contre les risques des crues exceptionnelles.

Enfin, beaucoup de progrès sont attendus, en matière de surveillance, contrôle et gestion de la sécurité. Les barrages sont maintenant inclus parmi les risques technologiques majeurs même si, statistiquement, ils se révèlent, en nombre annuel de victimes, très peu dangereux. La sûreté d'un ouvrage s'obtient, évidemment, dès la conception et la construction. Elle se gère ensuite par une surveillance et un contrôle régulier, précis, bien adapté, accompagné par un suivi et une interprétation en temps réel des informations recueillies. Le système mis en place en France dans ce domaine, par E.D.F., est exemplaire.

Rappelons que, dans notre pays, la législation est, à cet égard, très exigeante et comporte l'obligation de l'établissement de plans particuliers d'intervention (PPI) basés sur la modélisation de l'inondation provoquée par la rupture éventuelle de l'ouvrage. Des réglementations de ce type ont tendance, actuellement, à se généraliser un peu partout dans le monde.

Même si les barrages sont critiqués vis-à-vis de leurs inconvénients, on a vu que ces derniers pouvaient être, en grande partie, évités par de meilleures conceptions, méthodes de construction et modes de gestion. Malgré la diminution du nombre de sites utilisables, il est possible de créer encore de vastes réserves sans dégrader l'environnement et de façon économiquement viable. Il suffit pour cela de bien appliquer le savoir-faire et, un peu plus tard, les plus efficaces des techniques qui sont encore actuellement en développement.

\subsection{Eaux souterraines [6]}

Elles représentent des réserves considérables à plus ou moins grande profondeur. Elles constituent d'autre part un système de régulation extrêmement puissant du débit des cours d'eau, les nappes alluviales jouant, dans ce cadre, un rôle essentiel.

Il est en fait impossible, dans un concept de gestion globale du cycle de l'eau, de séparer eaux de surface et eaux souterraines.

A partir des connaissances actuelles il est tout à fait envisageable d'améliorer, de façon considérable, les techniques d'ex- 
ploitation et d'utilisation des aquifères. Leynaud [7] préconise l'utilisation beaucoup plus massive des stockages souterrains comme réservoirs-tampons. Collin et Margat [6] décrivent toute une série d'actions conseillables ou proposables pour une maîtrise active de ces aquifères. Leur démarche intellectuelle se place d'abord à l'échelle des bassins et des systèmes euxmêmes, où ils préconisent des séries d'aménagements de surface semblables à ceux envisagés par Leynaud, et qui sont à classer dans le domaine de la défense et de la restauration des sols et de celui de la gestion patrimoniale. Dans la zone d'appel des captages, ils proposent une gestion agricole adaptée (en particulier au niveau des engrais, des pesticides et des travaux, donc des itinéraires techniques), la création de stocks souterrains par infiltration d'eau de crue et même la mise en place de barrages souterrains pour bloquer de façon plus ou moins importante les inféroflux. La gestion peut être dynamique et être destinée à contrer les entrées d'eau marine. La gestion peut aussi être globale, au niveau d'une zone, en utilisant au mieux les portions d'aquiferes inondables et les zones palustres.

Partout où cela est possible et en particulier dans la zone de captage proprement dite, il faut réhabiliter les recharges artificielles et utiliser au mieux les structures existantes comme les fosses à granulats. Ces recharges peuvent être également une arme puissante contre certaines formes de pollution.

Dans les périmètres de protection, plusieurs types d'actions ont été essayés, essentiellement dans des zones karstiques. Rappelons également que la technique du barrage souterrain a été utilisée pour isoler des flux d'eau douce de l'eau marine. A ce niveau, diverses interventions sont possibles pour effectuer des corrections de qualité, soit par des actions de surface, soit par des injections dans le sous-sol.

Enfin, on peut agir au niveau des captages eux-mêmes. De nombreux procédés ont été envisagés et l'éventail des possibilités que propose l'industrie du traitement des eaux, est pratiquement illimité.

Rappelons, dans ce cadre, que plusieurs expériences ont été réalisées à l'étranger. En particulier, en Allemagne, on a utilisé à la fois les capacités de filtration, de réactions géochimiques naturelles et de traitement artificiel pour épurer, dans la nappe elle-même, des eaux polluées, plus ou moins chargées de matières nocives (dénitrification et déferrisation sur le site de Brochoï).

On peut également penser à utiliser les très importantes réserves situées dans les karsts en pompant momentanément à un débit supérieur au débit courant et en laissant ensuite la réserve se reconstituer en période de crue. [8]

Compte tenu de tout cela, on voit combien il est nécessaire de protéger efficacement les nappes des pollutions diffuses et concentrées.

En ce qui concerne les pollutions industrielles, il s'agit avant tout de volonté politique et d'investissements. Pour les pollutions agricoles, on a vu plus haut les voies de progrès possibles. Dans tous les cas il s'agit d'une dégradation d'un capital qu'on ne peut combattre efficacement que par des moyens économiques : taxation sans doute, mais aussi, à terme, intégration obligatoire des externalités dans les coûts.

Pour progresser dans le domaine de la gestion des nappes il faudra aussi développer le nombre et la qualité des mesures. La sophistication informatique et électronique n'est pas forcement la voie la plus efficace. Les temps de réaction étant ici relativement lents, le stockage et le transfert de l'information peuvent s'opérer par des moyens rustiques et peu coûteux.

\subsubsection{Aller chercher la ressource et la transporter}

Transporter l'eau en dehors du système hydrographique reste et restera toujours coûteux. Néanmoins on y est forcément amené quand il n'existe pas d'autres solutions.

Dans le domaine des transferts sous pression, de grands progrès sont en cours en ce qui concerne les régulations de la commande des vannes.

Clément et al. [9] ont décrit une méthode de régulation de l'alimentation en eau des métropoles urbaines par des conduites de grande longueur et de grand diamètre. Mise au point à la Sogreah, cette méthode reprend les raisonnements classiques des commandes par l'amont et par l'aval en profitant des avantages des deux solutions. Son application est rendue possible par la mise au point de régulateurs de grandes dimensions pouvant agir sur une adduction en limitant les pressions. Il s'agit là d'un élément des importants progrès en cours dans la conception des grands projets d'alimentation en eau.

L'utilisation de canaux reste toutefois le moyen fondamental pour transporter les très grands débits.

Sur le canal de Provence, un mode de gestion original lié à sa structure et au mode de distribution a été mis en place dès le départ. Il s'agit d'une gestion d'ensemble, obtenue grâce à une régulation dynamique, gérée par un logiciel qui agit, en temps réel, sur l'ensemble du système, en contrôlant les informations, en commandant les alarmes générales, en prévoyant les besoins, en régulant débits et volumes automatiquement, enfin en optimisant globalement le fonctionnement. Bien entendu, le système traite les données et informations pour en tirer, a posteriori, les bilans et statistiques nécessaires. [9]

Les dispositifs comme celui du canal de la Neste au pied des Pyrénées, représentent des réussites techniques incontestables. Bien entendu, construits et adaptés, au fil des années, aux circonstances géographiques, hydrologiques et sociales locales, ils ne peuvent être transposés tels quels. Ils constituent toutefois des modèles extrêmement intéressants, et ont permis la mise au point de méthodes de gestion et de régulation tout à fait transposables.

Parallèlement, la technique des machines hydrauliques progresse. Megnint (1992) a décrit, dans un rapport sur les turbinespompes, les caractéristiques et les applications de ces machines, développées au départ pour les centrales hydro-électriques, mais qui peuvent être utilisées dans des aménagements à buts multiples, où elles offrent des possibilités extrêmement intéressantes grâce à leur réversibilité. La gamme des matériels permet de couvrir des dénivelées allant de quelques mètres à $500 \mathrm{~m}$. Le potentiel d'utilisation de ces machines est considérable. Les liaisons interbassins deviennent évidemment, avec de tels équipements, beaucoup plus facilement envisageables. Des liaisons qui permettent des régulations de débit sans avoir à construire des barragesréservoirs, ont déjà été réalisées par des méthodes gravitaires.

Allant bien au-delà, les ingénieurs de la Compagnie Nationale du Rhône ont envisagé et étudié le soutien des étiages de la Loire et de la Seine à partir du Rhône. (C. Cottereau, 1992)

Après avoir constaté que le régime du Rhône d'une part et celui de la Loire et de la Seine d'autre part, étaient largement complémentaires en particulier en été, ils proposent l'installation d'un système de transfert entre la retenue de Péage de Roussillon sur le Rhône et celle de Grangent sur la Loire. Une fraction du débit pompé vers la Loire pourrait ensuite alimenter la Seine par une station de pompage de $10 \mathrm{~m}^{3} / \mathrm{s}$ utilisant le canal de Briare.

Ce genre de réalisation est parfaitement à la portée de la technique actuelle. Elle devrait, bien évidemment, être précé- 
dée d'études économiques et écologiques approfondies. Les principales objections seraient sans aucun doute d'ordre environnemental ou politique. L'expérience a prouvé que, dans un pays voisin, l'Espagne, un transfert important, du Tage vers le Ségura, a entraîné des vives polémiques, les usagers du bassin "prélevé" s'estimant dépouillés de leurs ressources en eau. De telles difficultés sont encore accrues quand les transferts ont lieu d'un pays à un autre.

Le transfert de l'eau devient maintenant possible par voie maritime. La Société du Canal de Provence (SCP) a déjà réalisé de tels transports [10].

La SCP qui dispose d'un potentiel de fourniture d'eau de 700 millions de $\mathrm{m}^{3} / \mathrm{an}$ et qui alimente déjà 60000 ha irrigués, 100 villes regroupant 1500000 habitants et de nombreuses industries, a mis en place des ouvrages principaux dont la longueur dépasse $270 \mathrm{~km}$ et qui aboutissent sur le littoral entre Marseille et Fos, ainsi que dans la région de Toulon. Une canalisation pouvant délivrer $3000 \mathrm{~m}^{3} / \mathrm{h}$, arrive en particulier au port pétrolier de Lavera, où le môle destiné au chargement de l'eau est accessible aux tankers d'un tonnage maximal de $100000 \mathrm{t}$.

De 1983 à 1990, plusieurs millions de $\mathrm{m}^{3}$ d'eau ont été ainsi exportés vers la région de Tarragone en Espagne et vers le sud de la Sardaigne, par des navires citernes de 48000 et $84000 \mathrm{~m}^{3}$ de capacité. Les prix de revient actuels assez élevés, de 20 à $25 \mathrm{~F} / \mathrm{m}^{3}$ pour des transports à $500 \mathrm{~km}$, sont susceptibles d'être sensiblement baissés pour des contrats pluriannuels de plusieurs millions de $\mathrm{m}^{3}$. Ils peuvent même, pour les distances inférieures à $1000 \mathrm{~km}$, devenir inférieurs à celui du dessalement, ce qui amène à envisager ce type de transfert pour alimenter des industriels et des villes.

L'extrême souplesse du transport maritime peut ainsi apporter une solution intéressante en cas d'arrêt ou de panne d'usine de dessalement ou de traitement, d'une sécheresse locale exceptionnelle, ou d'une pointe saisonnière, ou enfin en attendant la mise en place d'une structure de renforcement.

Le principal inconvénient, de cette méthode, à côté de son coût, est la mise en dépendance du consommateur vis-à-vis du fournisseur, surtout s'il est étranger.

\subsubsection{Traiter les eaux polluées - Dessaler l'eau de mer}

Les eaux polluées représentent une ressource importante qu'il faut essayer de valoriser au maximum. Il est évident que l'avenir de l'industrie du traitement des eaux est brillant.

Au niveau du traitement des eaux brutes, les normes et exigences de qualités, dans tous les pays du monde, deviennent graduellement plus sévères alors que parallèlement, les besoins en eau s'accroissent et que de multiples pollutions concentrées ou diffuses apparaissent au niveau de la ressource.

Le progrès est continu et le renouvellement des techniques, rapide. Malgré cela, les prix de traitement s'accroissent car les besoins en investissements sont énormes et les exigences de qualité toujours plus sévères.

Un des progrès récents, intéressant à citer, est l'utilisation des membranes d'ultrafiltration, qui permettent la création de petites unités de traitement (éventuellement transportables) efficaces et peu gourmandes en énergie. [11]

On a vu que le traitement ou le recyclage des eaux usées s'impose maintenant, presque systématiquement, et devient en bien des endroits, obligatoire. Or, il s'agit probablement d'un des domaines de la technique où les progrès scientifiques, la pluridisciplinarité et la valeur des recherches, sont les plus marqués bien que les spécialistes restent par tradition assez discrets en la matière. A côté des progrès continus et évidents au niveau des méthodes physiques, chimiques et biologiques, des avancées sont nécessaires pour permettre le contrôle, en toute période, de débits très variables d'effluents, pour faire face à des crises accidentelles, et enfin pour limiter au maximum les rejets de microorganismes pathogènes.

L'extension obligatoire, à terme, à toutes les agglomérations, d'installations de traitement de bonne qualité, pose également des problèmes financiers très difficiles, qui ne sont pas limités aux pays en développement.

Indiquons enfin que la mise au point de petites unités de traitement, très efficaces et bien adaptées à des maisons particulières ou à des immeubles collectifs de petite taille et fabriqués à bas prix en grande série, peut relancer dans les années à venir les discussions sur les avantages de l'assainissement individuel.

Les techniques de dessalement des eaux saumâtres ou de l'eau de mer ont progressé de façon considérable.

On distingue classiquement les procédés faisant intervenir les changements de phase (distillation-congélation, extraction par formation d'hydrates), les procédés agissant sur les liaisons chimiques (échange d'ions, extraction par solvants sélectifs), enfin les procédés utilisant les membranes (électrodyalise, osmose inverse, procédé osmo-ionique).

La distillation elle-même comporte de nombreuses variantes (apport de chaleur, compression de vapeur, distillation solaire...). Elle s'est beaucoup développée pour les moyennes et grandes capacités. Le prix de l'eau produite dépend étroitement de celui de l'énergie.

Il est possible qu'en combinant à la fois la production d'énergie et celle de chaleur à basse température (cogénération), l'on puisse dessaler l'eau de mer à des coûts de plus en plus bas. Il apparaît difficile néanmoins que, dans le cadre des progrès envisageables de cette technique, l'on arrive à moyen terme, à des prix de production suffisamment bas pour permettre l'irrigation sur des surfaces importantes.

Il est toutefois possible qu'en zone tropicale désertique, l'utilisation massive du rayonnement solaire direct puisse, dans de très grandes installations, conduire à des coûts sensiblement plus bas que ceux obtenus actuellement.

Depuis quelques années, et en parallèle, la technique par osmose inverse, utilisant des membranes de plus en plus efficaces, a permis des assainissements sensibles des prix de revient puisqu'on aboutit actuellement à des coûts de production de l'ordre de $1 \$$ le $\mathrm{m}^{3}$ permettant d'intéressantes installations d'alimentation de certaines îles, comme c'est le cas à Malte. (A. Riolo, J. Mangion, R. Gouzes, A. Guttierez, 1992)

Il est donc probable que, dans l'avenir, les progrès continus des techniques de dessalement amèneront à utiliser de plus en plus l'eau ainsi produite, dans toutes les zones où l'obtention d'eau douce d'origine naturelle est pratiquement impossible pour des raisons techniques, économiques ou d'environnement.

\subsubsection{Modifier le climat - La pluie provoquée}

L'idée de modifier localement le climat, en particulier par des techniques de pluie provoquée, a connu une certaine vogue, il y a une vingtaine d'années.

Quelques résultats positifs ont pu être obtenus, soit par la création de courants convectifs artificiels, soit par ensemencement de nuages dans une atmosphère déjà fortement humide.

Ces résultats très partiels, pour des coûts environnementaux ou économiques en général excessifs, n'ont pas donné lieu à des applications suivies. 
En fait, les quantités d'énergie à mettre en œuvre sont trop importantes et les connaissances de la dynamique de l'atmosphère sont encore trop limitées pour que l'on puisse, pour l'instant, envisager d'utiliser ces méthodes à une échelle significative. Les éventuels progrès dans ce domaine (s'ils sont possibles) demanderont sans doute de nombreuses années.

\section{III $\square$ PROGRÈS TECHNIQUE - SOCIO-ÉCONOMIE - GESTION PATRIMONIALE}

Le progrès technique $\mathrm{a}$, sauf révolution peu probable, un potentiel de croissance quasi-illimité du fait qu'il s'agit d'un processus auto-alimenté, soutenu par des tendances lourdes.

Cela ne veut évidemment pas dire qu'à court ou moyen termes, la technique puisse tout faire. Nous avons vu, au long de ces développements, l'étendue des limites qui séparent le savoir du pouvoir réaliser. Ces limites sont jusqu'à présent essentiellement socio-économiques ; les préoccupations d'éthique y prendront demain une part croissante. La réticence envers les transferts inter-bassins en est un bon exemple.

Une technique puissante donne souvent au décideur pressé la possibilité de résoudre rapidement et au moindre prix, un problème local, sans trop se soucier des coûts externes et des problèmes à long terme.

Nous pensons qu'il est possible d'utiliser beaucoup plus intelligemment les énormes possibilités de notre savoir-faire actuel dans le cadre de ce qu'on appelle maintenant couramment la gestion patrimoniale des ressources naturelles, c'est-àdire, dans le cas présent, des ressources en eau.

Le principe, on l'a vu, consiste à utiliser au maximum les cycles naturels en prélevant l'eau là où cela est nécessaire et en l'y rejetant, traitée au mieux pour que les écosystèmes continuent à fonctionner correctement et que l'utilisateur aval puisse s'approvisionner sans difficulté.

Cela conduit, bien évidemment, à envisager une telle gestion à l'échelle du bassin versant, en y intégrant les aquiferres intéressés. Cela veut dire aussi qu'il vaut mieux, au long d'une rivière, recycler au maximum et traiter les eaux rejetées que de créer des réserves tout en haut du bassin versant et transporter l'eau par tuyaux jusqu'aux villes situées le plus en aval.

Même si ce concept est un peu plus coûteux au départ, son application peut amener, à l'échelle d'une nation ou d'une région, et sur plusieurs générations, des économies considérables tout en assurant une sauvegarde évidente de l'environnement.

Sa mise en œuvre passe par un changement des mentalités, donc une éducation réorientée des citoyens, une amélioration des outils juridiques et fiscaux et surtout une refonte complète des méthodes de calcul économique.

Les intérêts immédiats en jeu sont malheureusement tels, que ce genre de changement ne s'obtiendra pas aisément ni rapidement.

On peut espérer que les progrès de la recherche économique et une pédagogie efficace visant à convaincre les décideurs, à l'échelle mondiale, permettront d'y parvenir.

On peut craindre à l'inverse que l'inertie des mentalités et la lourdeur des structures politico-financières en place, en retarde l'arrivée jusqu'à ce qu'une catastrophe, d'ampleur suffisante, amène ces décideurs à vraiment s'interroger.

Une telle catastrophe pourrait d'ailleurs être le changement climatique (réchauffement) dû à l'accroissement des taux de concentration des gaz à effet de serre dans l'atmosphère. [8], [12]
Les scientifiques et les techniciens qui aiment à rêver de l'avenir sont, par nature, des optimistes. Ils sont persuadés qu'on pourra à moyen terme gérer rationnellement, à toutes les échelles et dans l'esprit d'un développement durable à long terme, cette eau si totalement indispensable.

\section{Références bibliographiques}

N.B - Les publications concernant les applications du progrès technologique au domaine de l'eau sont multiples et quasiment innombrables. Les auteurs cités ne représentent donc qu'un tout petit échantillonnage de la masse totale des publications. Un large emprunt a été fait aux comptes rendus des Vingt Deuxièmes Journées de l'Hydraulique de la Société Hydrotechnique de France (SHF) sur l'Avenir de l'Eau et qui représentent, pour ce thème, une synthèse particulièrement intéressante.

[1] Margat J. et Tiercelin J. R. - L'eau en questions, Romillat, Paris, 1998. $301 \mathrm{p}$.

[2] Dourlens C. et Vidal. Naquet P.A. - La ville au risque de l'eau. L'Harmattan Logiques Sociales, Paris, 1992.

[3] JARRAUD et GELEYN - La prévision du temps. La recherche-Météo, $\mathrm{n}^{\circ} 201$, 1988.

[4] BASSAZ F. et SOMBroEK W. - Changements climatiques et production agricole, FAO - Rome, Polytechnica - Paris, 1997.

[5] ALLEN J. - Biosphère 2. The human experiment, Penguin Books Science, New-York, 1991.

[6] Margat J. et Collin J.J. - L'avenir de l'eau souterraine : la gestion active des aquiferes. SHF, I'Avenir de l'Eau, Vingt Deuxièmes Journées de l'Hydraulique, Paris, 1992.

[7] LeYNAUD G. - Méthodes et techniques alternatives ou complémentaires aux barrages de retenue pour la régularisation des ressources en eau. SHF, L'Avenir de I'Eau, Vingt Deuxièmes Journées de I'Hydraulique, Paris, 1992.

[8] Bakalowicz M. , Biscaldi R.,Mangin A., Marchet P., Marsaud B. et RICARD J. - Utilisation des réserves en eau d'un karst par pompage à gros débit. SHF, L'Avenir de I'Eau, Vingt Deuxièmes Journées de l'Hydraulique, Paris, 1992.

[9] Clement G., Valibouse B., Dartout P.Y.- Grands transferts d'eau en conduite, nouveaux principes de régulation. Nouveauté dans la régulation des grandes conduites d'adduction d'eau, SHF, l'Avenir de l'Eau, Vingt Deuxièmes Journées de l'Hydraulique, Paris, 1992.

[10] Masson G. - La société du Canal de Provence et le transport de l'eau par voie maritime. SHF, L'Avenir de l'Eau, Vingt Deuxièmes Journées de l'Hydraulique, Paris, 1992.

[11] Mandra V., Anselme C., Audic J.M., Mazounie P. - Désinfection d'eaux résiduaires urbaines par ultrafiltration. Courants, $n^{\circ} 20$, Paris, 1993.

[12] DUPLESSY J.C. et MOREL P.- Gros temps sur la planète, Odile Jacob, Paris, 1990.

[13] Cans R. - La bataille de l'eau. Le Monde Éditions, Paris, 1997.

[14] DuRAND D. et Tourasse P. - Progrès en cours et prévisibles en hydrologie et climatologie opérationnelles, SHF, L'Avenir de l'Eau, Vingt Deuxièmes Journées de l'Hydraulique, Paris, 1992.

[15] SERVAT E. et DezetTER A. - Évaluation des ressources en eau dans le Nord-Ouest de la Côte d'Ivoire. Extensions aux bassins versants non jaugés. SHF, L'Avenir de l'Eau, Vingt Deuxièmes Journées de l'Hydraulique, Paris, 1992. 